\title{
Cognitive and home environmental predictors of change in sugar-sweetened beverage consumption among adolescents
}

\author{
Nicole P. M. Ezendam ${ }^{1}$, Alexandra E. Evans ${ }^{2}$, Melissa H. Stigler ${ }^{2}$, Johannes Brug ${ }^{3}$ and Anke Oenema ${ }^{1}$ \\ ${ }^{1}$ Department of Public Health, Erasmus University Medical Center, PO Box 2040, 3000 CA Rotterdam, The Netherlands \\ ${ }^{2}$ School of Public Health, University of Texas, Austin, TX, USA \\ ${ }^{3}$ EMGO Institute, VU University Medical Center, Amsterdam, The Netherlands
}

(Received 3 December 2008 - Revised 8 September 2009 - Accepted 10 September 2009 - First published online 14 December 2009)

Sugar-sweetened beverage (SSB) consumption may increase risk for unnecessary weight gain. To develop interventions discouraging consumption, more insight is needed about cognitive and environmental predictors related to the decrease in SSB consumption. The present paper aims (1) to describe the relationship between potential cognitive determinants of change (attitudes, subjective norms, perceived behavioural control and intentions) and perceived environmental factors (family food rule and home availability of SSB) with changes in SSB consumption between baseline and 4-month follow-up and (2) to study whether the relationships between the environmental factors and SSB consumption are mediated by the cognitive determinants. Information on possible predictors and SSB intake at baseline and 4-month follow-up was provided by 348 Dutch adolescents (aged 12-13 years) through online questionnaires that were completed at school. Multilevel logistic regression and mediation analyses were used to determine direct and indirect associations between predictors and behaviour. The present results show that a high perceived behavioural control to decrease intake at baseline was associated with a decrease in consumption of SSB between baseline and follow-up $(\mathrm{OR}=0.53)$. Low availability and a stricter family food rule were associated with a decrease in SSB consumption between baseline and follow-up $(\mathrm{OR}=2.39,0.54)$. The association between availability and decrease in SSB consumption was for $68 \%$ mediated by perceived behavioural control to drink less. In conclusion, interventions to decrease SSB intake should focus on improving attitudes and perceived behavioural control to reduce intake, and on limiting home availability and stimulating stricter family food rules regarding SSB consumption.

\section{Adolescents: Determinants: Sugar-sweetened beverages: Soft drinks: Environment}

Sugar-sweetened beverage (SSB) intake has been associated with an increased risk for overweight and obesity ${ }^{(1,2)}$. High SSB intake is very common among adolescents ${ }^{(3-6)}$. Estimates range from half to one litre of soft drink consumption on average per day ${ }^{(3-5)}$ with a frequency of almost $5 \mathrm{~d}_{\text {per }}$ week $^{(6)}$. In the past decades, there has been an increase in SSB consumption among adolescents worldwide ${ }^{(5,7,8)}$. Therefore, to prevent the development of overweight and obesity among adolescents, decreasing the consumption of SSB is an important target in behavioural interventions. To be able to develop effective interventions aimed at lowering SSB intake, a detailed understanding of the determinants of this specific behaviour among adolescents is needed ${ }^{(9)}$. Specifically, more knowledge about the determinants of improving a behaviour (e.g. decreased SSB consumption) as opposed to determinants of a healthy behaviour (e.g. little SSB consumption) is needed.

The theory of planned behaviour (TPB) is one of the most widely used models in studying potential determinants of health behaviours, including dietary behaviours ${ }^{(10)}$. The theory postulates that a behaviour is predicted by an intention to engage in this behaviour and that this intention is determined by attitudes (perceived pros and cons of the behaviour), subjective norm (perception of important others' expectations regarding the behaviour) and perceived behavioural control (perceived difficulty to engage in the behaviour) ${ }^{(11)}$. Some cross-sectional and one longitudinal study has used the TPB to explore possible determinants of SSB intake ${ }^{(4,5,12-16)}$. For SSB consumption, consistent relationships were found with attitude, subjective norm and intention, whereas the relationship between perceived behavioural control and SSB consumption was less consistent.

The Environmental Research Framework for Weight Gain Prevention is a framework that posits clear mediation and interaction between cognitive and environmental determinants of energy balance behaviours ${ }^{(17)}$. Environmental influences are hypothesised to influence behaviour either directly or indirectly via cognitions. To increase our understanding of the mechanisms between environmental and cognitive determinants, the mediating role of cognitive factors in the relationship between environmental factors and behaviour is currently an important target in behavioural research. The home food environment may be of specific importance for adolescents, since adolescents consume a lot of SSB at home ${ }^{(18)}$. Both the availability of SSB and the family food rules for consumption of SSB provide specific conditions that might influence SSB intake at home. Therefore, availability

Abbreviations: SSB, sugar-sweetened beverage; TPB, theory of planned behaviour.

* Corresponding author: Nicole P. M. Ezendam, fax +31 10 7044724, email n.ezendam@erasmusmc.nl 
(i.e. the physical environment) and family food rules (i.e. a political factor) have been studied as possible correlates of SSB consumption in some studies (mostly cross-sectional; one longitudinal) ${ }^{(4,14-16,19,20)}$, providing evidence that such factors may indeed be of additional importance. Studies are now needed that investigate potential cognitive and environmental factors together, and preferably in longitudinal research ${ }^{(21)}$.

The purpose of the present study was to use longitudinal data to identify cognitive and environmental predictors of changes in SSB consumption among adolescents over a 4-month period. The cognitive measures were derived from the TPB and included attitudes, subjective norms, perceived behavioural control and intention toward reducing the intake of SSB. The environmental variables included availability and the family food rule at home regarding SSB consumption. In addition, we explored whether the effect of the environmental factors on SSB consumption was mediated by the cognitive factors (attitudes, subjective norms, perceived behavioural control and intentions) in the pathway between environmental factors (availability and family food rule) and change in behaviours (intake of SSB). The conceptual framework is shown in Fig. 1.

\section{Methods}

The present paper used longitudinal data of the FATaintPHAT intervention study for secondary analysis.

FATaintPHAT is an intervention study that targets adolescents to prevent excessive weight gain through changes in energy balance-related behaviours. The study design and intervention are described thoroughly elsewhere ${ }^{(22)}$. In the present study, control group data regarding SSB intake and determinants from baseline and 4-month follow-up were used.

\section{Study design and participants}

Twenty secondary schools in Rotterdam and surrounding areas provided consent to participate in the FATaintPHAT study of which nine control schools were randomly assigned to the no intervention control group. Data from this group were used for the present study. Approximately, four classes per school were randomly selected to participate in the study. During school year 2006-2007, baseline (from November till February) and 4-month follow-up (from March till June) measurements were conducted among adolescents aged 12-13 years (first grade). In The Netherlands, schools for secondary education vary by educational level, ranging from lower vocational to university preparatory. Schools varying from vocational level up to university preparatory level participated in the

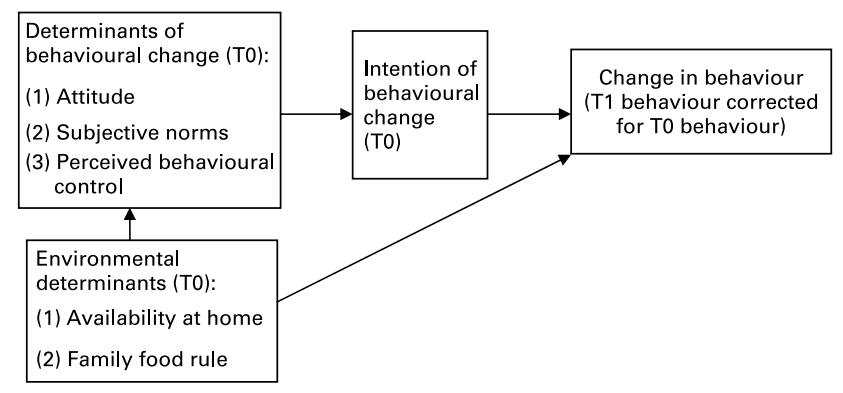

Fig. 1. Conceptual framework. present study. The study sample consisted of 398 students. The response rate was $56 \%$.

\section{Procedure}

At baseline and follow-up, electronic, self-administered questionnaires were administered to the students and used to assess intake of SSB, students' personal cognitions and perceived environmental factors. Questionnaires were administered during a classroom lesson (about $45 \mathrm{~min}$ ) under supervision of a research assistant. To increase participation rates, MP3 players were raffled at the end of the study among students who had completed all measurements.

\section{Measures}

Sugar-sweetened beverage intake. SSB were defined as carbonated soda, non-carbonated sugar-sweetened drinks and sport drinks. Frequency ('on how many days of the last $7 \mathrm{~d}$ did you drink SSB?') and quantity ('on a day you drank SSB, how much did you consume on average on a single day?') were assessed; answer categories were number of glasses $(200 \mathrm{ml})$, cans $(333 \mathrm{ml})$ and bottles $(500 \mathrm{ml})$ of SSB. Total daily SSB consumption was calculated as the average intake in millilitres per day. The FFQ that we used is based on a validated questionnaire ${ }^{(23,24)}$ and is frequently applied for assessing SSB intake among adolescents ${ }^{(5,25)}$. The 10-d test-retest reliability was $r \quad 0 \cdot 59^{(26)}$. Because normality checks showed that the intake was not normally distributed, we dichotomised SSB consumption into more than $400 \mathrm{ml}$ (1) $v$. equal or less than $400 \mathrm{ml}$ (0) per day, according to the median value of the dataset. This value also represents an often recommended maximum intake of SSB and provides therefore a meaningful cut-off point.

Individual cognitions and environmental factors. Cognitions specific for drinking less SSB according to the TPB (attitude, subjective norm, perceived behavioural control and intention) were assessed on five-point bipolar scales. General attitude toward drinking less SSB was measured with one item (e.g. for me drinking less SSB is very bad $(-2)$ - very good $(+2)$ ). Parental subjective norm was assessed with one item (e.g. do you think your parents want you to drink less SSB? certainly yes $(+2)$ - certainly not $(-2)$ ). Perceived behavioural control was assessed with two items covering the dimensions easy/difficult (e.g. do you think it is difficult or easy to drink less SSB? very difficult $(-2)$ - very easy $(+2)$ ) and the likelihood of succeeding (e.g. do you think you will succeed in drinking less SSB if you want to? certainly yes $(+2)$ - certainly not $(-2))$. These two variables were combined into one construct perceived behavioural control (Cronbach's $\alpha$ was 0.74$)$. Intention to change the behaviour was assessed with one item (e.g. do you intend to drink less SSB in the upcoming year? certainly yes $(+2)-$ certainly not $(-2)$ ). All individual cognitions were dichotomised because of the skewed distributions, where -2 till 0 were coded as 0 and +1 till +2 was coded as 1 .

Perceived home availability and the family food rule were assessed using five-point bipolar items. Availability at home was assessed with one item (e.g. are there SSB available at home? always $(+2)$, almost always $(+1)$, sometimes $(0)$, seldom $(-1)$ and never $(-2))$. This item was dichotomised as -2 till 0 as 0 and +1 till +2 as 1 . The family food rule 
was assessed with one item (e.g. are you allowed to drink as much SSB as you like at home? always (-2), almost always $(-1)$, sometimes $(0)$, seldom $(+1)$ and never $(+2)$ ). This item was dichotomised as -2 till -1 as 0 (liberal) and 0 till +2 as 1 (strict).

Demographics. Questions on age (how old are you? 11-18 or older), sex (are you a boy or a girl? boy; girl), educational level (indicate what level of education you attend (low vocational; higher vocation; middle; pre-university; pre-university plus)) and ethnicity (assessed with three items: what is your country of birth? what is your mother's country of birth? what is your father's country of birth) were included in the questionnaire. Respondents' ethnicity was categorised as either Dutch (both parents are born in the Netherlands) or non-Dutch according to Statistics Netherlands ${ }^{(27)}$.

\section{Analyses}

Respondents with missing data on the potential determinants or intakes at baseline and/or at follow-up were deleted from the analyses ( $n$ 36). $Z$-scores (value minus the mean of all values divided by the standard deviation) for the intakes were calculated, and outliers above $z$-score $3.29(P<0 \cdot 001$, two-tailed test) were recoded as missing ( $n$ 14). In addition, SSB intake levels above an average of 3 litres per day were truncated at 3 litres ( $n$ 20).

Multilevel logistic regression analyses were performed using MlwiN 2.02. A two-level structure was used to take the nesting structure of students within schools into account ${ }^{(28)}$. We fitted a model with the intercept and the baseline intake variable to examine the significance of the between-schools variance in changes in intake between schools. Significance was calculated with the one-tailed Wald statistic following a $\chi^{2}$ distribution with one degree of freedom. Significance of variance would indicate that the SSB intake clusters within schools. However, the between-school variance (random intercept) was 0.000 and consequently multilevel analyses were not required. Thus, we continued the regression analyses in SPSS 15.0 (SPSS Inc., Chicago, IL, USA).

We fitted several regression models ${ }^{(29)}$. First, we fitted a model with potentially confounder variables sex, age and ethnicity and baseline intake. Secondly, we added attitude, subjective norm and perceived behavioural control to the model. This model shows the significance of the more distal TPB predictors on change in intake. Thirdly, we added the most proximal TPB predictor intention to the model to examine whether intention was significantly related to change in intake after correcting for other predictors of the TPB and confounders. Fourthly, we added availability and family food rule to the model to investigate whether these factors were associated with change in intake after correcting for cognitive factors. Fifthly, we removed the TPB variables from the model to be able to describe the association of the environmental factors and intake, only corrected for the baseline intake and background variables. The models with time 2 behaviour as dependent variable and time 1 behaviour as covariate allow for a prediction of change in behaviour over the 4-month period ${ }^{(30,31)}$. So, an OR larger than one reflects an increased likelihood of having changed to the higher intake group, and thus a decreased likelihood that a high score on the determinant predicts a decrease in intake from $>400 \mathrm{ml}$ to $\leq 400 \mathrm{ml}$, while an OR lower than one reflects an increased likelihood that a high score on the determinant predicts a decrease in intake from $>400 \mathrm{ml}$ to $\leq 400 \mathrm{ml}$.

Mediation analysis following the product-of-coefficients test as described by McKinnon ${ }^{(32)}$ was used to see whether cognitive variables (at baseline) mediated the relation between the environmental factors (at baseline) and change in intake (intake at follow-up adjusted for baseline intake). Separate analyses were performed for the environmental factors availability and family food rules. An effect was said to be mediated if (1) the environmental variable had a statistically significant effect on the hypothesised mediator (cognitive variables; $a$ coefficient); (2) the hypothesised mediator (the cognitive variable) was associated with the outcome (change in SSB consumption) after controlling for the environmental variable ( $b$ coefficient); (3) the mediated effect (product of the $a$ and $b$ coefficients) was statistically significant. Because the product of two coefficients is not normally distributed, we used bootstrapping to determine the significance of the mediated effect $^{(32)}$. With bootstrap, the empirical distribution of the data is used to determine the confidence limits around the estimate of the mediated effect. A sample with replacement was drawn 1000 times from the original study sample. In each new sample, the mediated effect, $a \times b$, was estimated. The mediated effect estimated in each bootstrap sample was used to form a bootstrap distribution of the mediated effect estimates, and $95 \%$ CI were obtained from the bootstrap distribution (the $2 \cdot 5$ th and $97 \cdot 5$ th percentiles) ${ }^{(32)}$. Percentagemediated effect was calculated as $a b / c$, where $c$ is the coefficient in the relationship between predictor (availability or family food rules) and outcome (change in SSB). All statistical models employed in these analyses were adjusted for a set of covariates, namely sex, educational level, ethnicity and baseline intake. Mediation analyses were performed with S-PLUS 6.0 (Insightful, Inc., Seattle, WA, USA).

The present study was conducted according to the guidelines laid down in the Declaration of Helsinki, and all procedures involving human subjects were approved by the Medical Ethics Committee of the Erasmus University Medical Center. Written informed consent was obtained from all students and parents.

\section{Results}

Approximately, $73 \%$ of the study participants were Dutch and $51 \%$ attended higher secondary schools (Table 1). Individual cognitions regarding decreasing intake of $\mathrm{SSB}$, food rule and perceived availability of SSB are described in Table 2,

Table 1. Demographic characteristics of the participating students (Netherlands; $12-13$ years) at baseline (2006-2007)

\begin{tabular}{lc}
\hline & Students $(n$ 348) \\
\hline Sex (\% boys) & 47.7 \\
Ethnicity (\% Dutch) & 72.7 \\
Age at baseline (years) & 12.6 \\
Mean & 0.58 \\
SD & 50.6 \\
School level (\% high level $\left.{ }^{*}\right)$ & \\
\hline
\end{tabular}

*High school level is pre-university and pre-university plus (Havo/VWO). 
Table 2. Description of cognitive and environmental variables and intake levels for sugar-sweetened beverages (SSB) consumption of study participants at baseline ( $n$ 348, 2006-2007; Netherlands, 12-13 years)

\begin{tabular}{|c|c|}
\hline & Percentage of students \\
\hline \multicolumn{2}{|l|}{ Individual cognitions } \\
\hline \multicolumn{2}{|l|}{ Attitude to drink less } \\
\hline Negative/neutral & 53.7 \\
\hline Positive & $46 \cdot 3$ \\
\hline \multicolumn{2}{|l|}{ Parental subjective norm to drink less } \\
\hline Negative/neutral & $78 \cdot 7$ \\
\hline Positive & $21 \cdot 3$ \\
\hline \multicolumn{2}{|l|}{ Perceived behavioural control to drink less } \\
\hline Negative/neutral & $30 \cdot 2$ \\
\hline Positive & $69 \cdot 8$ \\
\hline \multicolumn{2}{|l|}{ Intention to drink less } \\
\hline Negative/neutral & $77 \cdot 0$ \\
\hline Positive & 23.0 \\
\hline \multicolumn{2}{|l|}{ Environment } \\
\hline \multicolumn{2}{|l|}{ Availability at home } \\
\hline Never/seldom/sometimes & $24 \cdot 1$ \\
\hline Almost always - always & $75 \cdot 9$ \\
\hline \multicolumn{2}{|l|}{$\begin{array}{l}\text { Family food rule (allowed to drink } \\
\text { as much SSB as liked) }\end{array}$} \\
\hline Non-restrictive (almost always/always) & $47 \cdot 1$ \\
\hline $\begin{array}{l}\text { Neutral/restrictive (never/ } \\
\text { seldom/sometimes) }\end{array}$ & $52 \cdot 9$ \\
\hline \multicolumn{2}{|l|}{ Behaviour } \\
\hline \multicolumn{2}{|l|}{ SSB consumption } \\
\hline Baseline: intake > $400 \mathrm{ml}$ per day & $45 \cdot 1$ \\
\hline Follow-up: intake $>400 \mathrm{ml}$ per day & $48 \cdot 3$ \\
\hline
\end{tabular}

showing that $23 \%$ indented to drink less SSB in the future. In approximately $76 \%$ of the homes of the students, SSB were always or almost always available. About half of the students (47\%) were allowed to drink as much as they wanted to drink. Almost half of the adolescents drank $400 \mathrm{ml}$ or more per day. Approximately, $18 \%$ of the students decreased their intake from $>400$ to $\leq 400 \mathrm{ml}$ per day, and $14 \%$ of the students increased their intake.

Table 3 shows the results of the stepwise logistic regression analysis on change in intake categories of SSB (more than $400 \mathrm{ml}$ or equal or less than $400 \mathrm{ml}$ ). Being a girl was associated with a lower likelihood for an increase in consumption. Additionally, a positive perceived behavioural control and a positive attitude to decrease SSB consumption were related to a decrease in consumption over time (model 2). This relationship became non-significant for attitude, but remained significant for perceived behavioural control when intention, food rule and perceived availability were added to the model (models 3 and 4). In model 4, lower home availability and more restrictions on consumption were associated with a decrease in consumption over time. No significant relations with change in SSB consumption were found for education, ethnicity, subjective norms and intentions. Perceived availability and food rule were directly associated with intention after controlling for baseline and background variables (model 5).

The relationship between availability and change in SSB consumption was mediated by perceived behavioural control to decrease intake (top of Fig. 2). Of the association between availability and change in SSB intake, $68 \%$ was mediated by perceived behavioural control. So, low availability is associated with high perceived behavioural control to decrease intake and this is associated with a decrease in intake. The relationship between availability or food rule and change in SSB consumption was not significantly mediated by any other cognition (bottom of Fig. 2).

\section{Discussion \\ Main findings}

The present study is one of the few studies that examined potential cognitive determinants of decreasing SSB intake as well as family food rules and perceived home availability of SSB as predictors of change in SSB consumption using longitudinal data. The present results show that adolescents with a high perceived behavioural control and more positive attitudes to decrease SSB intake were more likely to decrease their intake. In addition, adolescents reporting low availability of SSB at home and a family food rule, which restricted them to drink as much SSB as they liked, were more likely to decrease their intake as compared with students with high

Table 3. Multilevel stepwise logistic regression of change in sugar-sweetened beverages consumption on hypothesised predictors (cognitions and environmental factors; $n$ 348; Netherlands; year 2006-2007; age 12-13 years)

(Odds ratios and $95 \%$ confidence intervals)

\begin{tabular}{|c|c|c|c|c|c|c|c|c|c|c|}
\hline & \multicolumn{2}{|c|}{ Model $1\left(R^{2} 24 \%\right) \dagger$} & \multicolumn{2}{|c|}{ Model $2\left(R^{2} 28 \%\right)$} & \multicolumn{2}{|c|}{ Model $3\left(R^{2} 28 \%\right)$} & \multicolumn{2}{|c|}{ Model $4\left(R^{2} 33 \%\right)$} & \multicolumn{2}{|c|}{ Model $5\left(R^{2} 31 \%\right)$} \\
\hline & OR & $95 \% \mathrm{Cl}$ & OR & $95 \% \mathrm{Cl}$ & OR & $95 \% \mathrm{Cl}$ & OR & $95 \% \mathrm{Cl}$ & OR & $95 \% \mathrm{Cl}$ \\
\hline Baseline intake & $5 \cdot 52^{*}$ & $3.45,8.83$ & $4 \cdot 38^{*}$ & $2 \cdot 67,7 \cdot 19$ & $4 \cdot 22^{*}$ & $2.56,6.95$ & $3 \cdot 36^{*}$ & $1.99,5.66$ & $4 \cdot 03^{*}$ & $2 \cdot 46,6 \cdot 61$ \\
\hline Sex (girls $v$. boys) & $0.57^{\star}$ & $0.35,0.91$ & $0.58^{*}$ & $0.36,0.95$ & $0.58^{*}$ & $0.36,0.94$ & $0.51^{*}$ & $0.31,0.85$ & $0.49^{\star}$ & $0.30,0.81$ \\
\hline Education (high v. low) & 0.82 & $0.51,1.31$ & 0.80 & $0.49,1.29$ & 0.77 & $0.48,1.26$ & 0.80 & $0.49,1.33$ & 0.83 & $0.51,1.35$ \\
\hline Ethnicity (non-Dutch $v$. Dutch) & 0.90 & $0.53,1.52$ & 0.79 & $0.44,1.40$ & 0.79 & $0.44,1.41$ & 0.82 & $0.45,1.51$ & 0.93 & $0.54,1.62$ \\
\hline Attitude (high $v$. low) & & & $0.60^{*}$ & $0.36,0.98$ & 0.64 & $0.38,1.07$ & 0.65 & $0.38,1.12$ & & \\
\hline Subjective norm (high $v$. low) & & & 0.78 & $0.41,1.49$ & 0.82 & $0.42,1.59$ & 0.88 & $0.44,1.74$ & & \\
\hline PBC (high $v$. low) & & & $0.44^{*}$ & $0.25,0.76$ & $0.45^{\star}$ & $0.25,0.80$ & $0.53^{*}$ & $0.30,0.97$ & & \\
\hline Intention (high $v$. low) & & & & & 0.73 & $0.40,1.35$ & 0.86 & $0.45,1.62$ & & \\
\hline Availability (high v. low) & & & & & & & $2 \cdot 39^{*}$ & $1 \cdot 27,4 \cdot 51$ & $2 \cdot 59^{\star}$ & $1.40,4.77$ \\
\hline $\begin{array}{l}\text { Family food rules } \\
\text { (restrictive } v \text {. non-restrictive) }\end{array}$ & & & & & & & $0.54^{*}$ & $0.32,0.91$ & $0.49^{*}$ & $0.29,0.81$ \\
\hline
\end{tabular}

$R^{2}$, percentage variance explained; PBC, perceived behavioural control.

${ }^{*}$ Significant predictors.

†Baseline intake and sex together explain $23 \%$ of the variance. 


\begin{tabular}{llll}
$\begin{array}{l}\text { Availability } \\
\text { baseline }\end{array}$ & Cognition at baseline & $b$ & $a b(95 \% \mathrm{Cl})$ \\
\hline$a$ & Attitude & -0.591 & $-0.005(-0.385,0.350)$ \\
\hline 0.009 & $\begin{array}{l}\text { Subjective norm } \\
\text { Follow-up adjusted for baseline }\end{array}$ \\
0.308 & $\begin{array}{l}\text { Perceived behavioural control } \\
-1.317\end{array}$ & -0.607 & $0.800(0.047,1.941)$ \\
-0.432 & Intention & -0.443 & $0.191(-0.161,0.669)$ \\
\hline
\end{tabular}

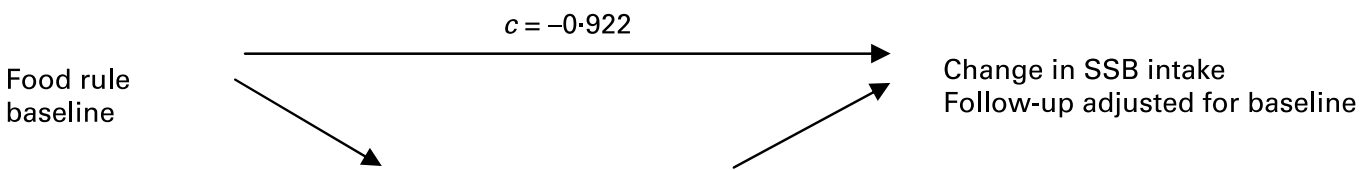

\begin{tabular}{llll}
\hline$a$ & Cognition at baseline & \multicolumn{1}{l}{$b$} & \multicolumn{1}{l}{$a b(95 \% \mathrm{CI})$} \\
\hline 0.711 & Attitude & -0.440 & $-0.313(-0.816,0.052)$ \\
0.897 & Subjective norm & 0.009 & $0.008(-0.654,0.612)$ \\
0.265 & Perceived behavioural control & -0.768 & $-0.204(-0.783,0.166)$ \\
0.967 & Intention & -0.354 & $-0.342(-1.120,0.226)$ \\
\hline
\end{tabular}

Fig. 2. Mediation analyses of cognitions to drink less sugar-sweetened beverages (SSB) in the association between availability (top of the figure)/food rule (bottom of the figure) and intake ( $n$ 348; Netherlands; year 2006-2007; age 12-13 years); $a, b$ and $c$ are $\beta$-coefficients.

availability and a liberal rule. The association between availability and decrease in SSB consumption was almost completely mediated by perceived behavioural control, which means that most of the effects of low availability on decrease in intake are explained by perceived behavioural control, i.e. children feel confident in reducing their SSB consumption when the availability of SSB is limited.

\section{Explanation and interpretation of results}

Literature on cognitive determinants of SSB consumption shows that attitudes, subjective norm and intentions of consuming SSB are related to intake ${ }^{(4,5,12,13,15,19)}$. The relationship between perceived behavioural control and SSB consumption is unclear ${ }^{(4,5,12,13,16,19)}$. We found significant relationships for attitudes and perceived behavioural control with changes in intake, but not for subjective norm and intention. Differences in results among different studies might be explained by different types of determinants that were measured as well as differences in study designs and the ways the potential determinants were assessed. We assessed determinants of behavioural change, while other studies measured determinants of low intake or situational intake. In addition, the design of the present study was longitudinal, whereas the designs of most other studies (except ${ }^{(4)}$ ) were cross-sectional. This difference may reveal differential associations, because longitudinal outcomes represent prediction and possibly causality, while cross-sectional studies only provide information on association. We were not able to test differences in associations between cross-sectional and longitudinal associations within the present study because of the way we assessed the cognitive factors (i.e. as related to change in behaviour).

Regarding possible physical and political environmental determinants, the present results showed that higher availability at home and a less strict family food rule were related to an increase in SSB intake. No other studies on possible determinants of change in intake have been published, but we could compare the present results with studies on possible determinants of intake. In that case, the present results are in accordance with most previous research ${ }^{(4,12,13,16)}$. However, one study showed that availability was not related in girls, and family food rules were not related with intake in boys and girls ${ }^{(16)}$. Furthermore, higher availability at home has been shown to be positively related to higher perceived behavioural control to drink soda ${ }^{(12,13)}$. In accordance, we found that higher home availability is related to a lower perceived behavioural control to drink less SSB. In addition, we found that perceived behavioural control mediates the relationship between availability and intake. The relationship between availability and perceived behavioural control indicates that one could influence an adolescents' perceived behavioural control by altering the home availability, which might add greatly to a direct target of increasing the perceived behavioural control of an adolescent. We did not find any mediation of cognitions in the relationship between family 
food rules and SSB intake. This confirms previous research where more strict parental practices were related to less SSB consumption, but this effect was largely unmediated by social cognitions ${ }^{(4)}$. The direct association between high perceived behavioural control, high home availability and a strict parental rule, and intake indicate that in future interventions to decrease SSB consumption, these cognitive and environmental factors should be targeted. The mediation between availability and intake by perceived behavioural control emphasises the important role of home availability and stresses the importance of targeting home availability in future interventions. So, the present results suggest an important role for parents. Although adolescents are becoming more autonomous while growing up, the influence of the parents is still important.

\section{Limitations}

The first limitation of the present study regards the follow-up period that we used with regard to the stability of the possible determinants. The time lag between baseline and follow-up may have been too long, thus obscuring relevant relationships in shorter periods of time. The optimal follow-up period is related to stability of determinants within students over time. We could not find any research regarding the stability of determinants of behavioural change. Individual cognitions of behaviours are considered stable over a longer time period $^{(33)}$. However, determinants of behavioural change might be more susceptible to variations due to, e.g. health eating campaigns, obscuring the relations that we examined.

The second limitation is that both behaviour and determinants are self-reported, which might lead to recall bias and social desirable answers. Overweight girls tend to underreport mainly foods high in energy density and low in nutrients, like $\mathrm{SSB}^{(34)}$. However, this does not necessarily mean that change in intake over a 4-month period is prone to bias as well.

The third limitation is that we dichotomised the outcome variable, SSB intake, because of its skewed distribution. The dichotomised outcome is probably a less sensitive outcome measure than a continuous one, because you look at change between intake categories (more or equal/less than $400 \mathrm{ml}$ ) and not at any change in intake, resulting in an underestimation of the change in intake. Only when the changes in intake were present about the $400 \mathrm{ml}$ turning point, the dichotomised outcome would be more sensitive. Examination of our data shows that this latter situation is not applicable in the present study. Despite the weaker dichotomised outcome measure, we did find significant relations, indicating that these predictors are strong predictors of change in intake.

A fourth limitation is that we assessed the cognitive factors with one- (attitude, subjective norm and intention) or two-item measures (perceived behavioural control). These measures may have only covered part of the larger construct. For example, one's attitude toward SSB intake may not only include an evaluation in terms of good and bad, but also pleasant/unpleasant. However, it is likely that the subtle differences between different evaluations of the same construct are not acknowledged by adolescents. The age group and the classroom administration of the questionnaire forced us to restrict the number of questions as much as possible.
A fifth limitation is that we cannot rule out any seasonal influences on SSB intake, leading to differences in intakes between the measurements. However, because we did not include the summer period in the present study, large seasonal variations are not likely.

\section{Conclusions}

The present longitudinal study provides important new insights into cognitive and environmental predictors of changes in SSB consumption. Both personal cognitions (attitude and perceived behavioural control) and factors in the home environment (availability and parental rules) appear to be important for adolescents' changes in SSB consumption. The results suggest that interventions to decrease SSB intake should focus on improving attitudes and perceived behavioural control to reduce intake, and on limiting home availability and stimulating stricter family food rules regarding SSB consumption.

\section{Acknowledgements}

The present study was funded by a grant from ZonMw (no. 62200020), The Netherlands Organization for Health Care Research and Development, and was supported by the Vereniging Trustfonds Erasmus Universiteit.

The authors declare no conflict of interests. N. P. M. E., A. E. E., A. O. and J. B. contributed to the design of the study; N. P. M. E., A. E. E., M. H. S., A. O. and J. B. contributed to the analyses and to the drafting of the manuscript.

\section{References}

1. Newby PK (2007) Are dietary intakes and eating behaviors related to childhood obesity? A comprehensive review of the evidence. J Law Med Ethics 35, 35-60.

2. Swinburn BA, Caterson I, Seidell JC, et al. (2004) Diet, nutrition and the prevention of excess weight gain and obesity. Public Health Nutr 7, 123-146.

3. Haerens L, De Bourdeaudhuij I, Maes L, et al. (2007) The effects of a middle-school healthy eating intervention on adolescents' fat and fruit intake and soft drinks consumption. Public Health Nutr 10, 443-449.

4. de Bruijn GJ, Kremers SP, de Vries H, et al. (2007) Associations of social-environmental and individual-level factors with adolescent soft drink consumption: results from the SMILE study. Health Educ Res 22, 227-237.

5. van der Horst K, Timperio A, Crawford D, et al. (2008) The school food environment associations with adolescent soft drink and snack consumption. Am J Prev Med 35, 217-223.

6. Vereecken CA, De Henauw S \& Maes L (2005) Adolescents' food habits: results of the Health Behaviour in School-aged Children survey. Br J Nutr 94, 423-431.

7. Popkin BM \& Nielsen SJ (2003) The sweetening of the world's diet. Obes Res 11, 1325-1332.

8. French SA, Lin BH \& Guthrie JF (2003) National trends in soft drink consumption among children and adolescents age 6 to 17 years: prevalence, amounts, and sources, 1977/1978 to 1994/ 1998. J Am Diet Assoc 103, 1326-1331.

9. Bartholomew LK, Parcel GS, Kok G, et al. (2006) Planning Health Promotion Programs. San Francisco, CA: Jossey-Bass. 
10. Baranowski T, Cullen KW, Nicklas T, et al. (2003) Are current health behavioral change models helpful in guiding prevention of weight gain efforts? Obes Res 11, Suppl, 23S-43S

11. Glanz K, Rimer BK \& Lewis FM (editors) (2002) Health Behavior and Health Education: Theory, Research and Practice. San Francisco, CA: Jossey-Bass.

12. Kassem NO, Lee JW, Modeste NN, et al. (2003) Understanding soft drink consumption among female adolescents using the Theory of Planned Behavior. Health Educ Res 18, 278-291.

13. Kassem NO \& Lee JW (2004) Understanding soft drink consumption among male adolescents using the theory of planned behavior. J Behav Med 27, 273-296.

14. Grimm GC, Harnack L \& Story M (2004) Factors associated with soft drink consumption in school-aged children. $J$ Am Diet Assoc 104, 1244-1249.

15. Bere E, Glomnes ES, te Velde SJ, et al. (2008) Determinants of adolescents' soft drink consumption. Public Health Nutr 11, 49-56.

16. Haerens L, Craeynest M, Deforche B, et al. (2008) The contribution of psychosocial and home environmental factors in explaining eating behaviours in adolescents. Eur J Clin Nutr 62, 51-59.

17. Kremers SP, de Bruijn GJ, Visscher TL, et al. (2006) Environmental influences on energy balance-related behaviors: a dual-process view. Int J Behav Nutr Phys Act 3, 9.

18. Savige G, Macfarlane A, Ball K, et al. (2007) Snacking behaviours of adolescents and their association with skipping meals. Int J Behav Nutr Phys Act 4, 36.

19. De Bourdeaudhuij I \& Van Oost P (2000) Personal and family determinants of dietary behaviour in adolescents and their parents. Psychol Health 15, 751-770.

20. Campbell KJ, Crawford DA \& Ball K (2006) Family food environment and dietary behaviors likely to promote fatness in 5-6 year-old children. Int J Obes (Lond) 30, 1272-1280.

21. Brug J, van Lenthe FJ \& Kremers SP (2006) Revisiting Kurt Lewin: how to gain insight into environmental correlates of obesogenic behaviors. Am J Prev Med 31, 525-529.

22. Ezendam NP, Oenema A, van de Looij-Jansen PM, et al. (2007) Design and evaluation protocol of "FATaintPHAT", a computer-tailored intervention to prevent excessive weight gain in adolescents. BMC Public Health 7, 324.

23. Van Assema P, Brug J, Ronda G, et al. (2001) The relative validity of a short Dutch questionnaire as a means to categorize adults and adolescents to total and saturated fat intake. J Hum Nutr Diet 14, 377-390.

24. Van Assema P, Brug J, Ronda G, et al. (2002) A short Dutch questionnaire to measure fruit and vegetable intake: relative validity among adults and adolescents. Nutr Health 16, 85-106.

25. Singh AS, Chin APMJ, Kremers SP, et al. (2006) Design of the Dutch Obesity Intervention in Teenagers (NRG-DOiT): systematic development, implementation and evaluation of a school-based intervention aimed at the prevention of excessive weight gain in adolescents. BMC Public Health 6, 304.

26. van der Horst K, Oenema A, Te Velde SJ, et al. (2009) Gender, ethnic and school type differences in overweight and energy balance-related behaviours among Dutch adolescents. Int $J$ Pediatr Obes 1-10.

27. Statistics Netherlands, www.cbs.nl (accessed 1 September 2008).

28. Twisk JW (2006) Applied Multilevel Analysis. Cambridge: Cambridge University Press.

29. Tabachnick BG \& Fidell LS (2007) Using Multivariate Statistics, 5th ed. Needham Heights, MA: Pearson Education.

30. Twisk J \& Proper K (2004) Evaluation of the results of a randomized controlled trial: how to define changes between baseline and follow-up. J Clin Epidemiol 57, 223-228.

31. Neumark-Sztainer DR, Wall MM, Haines JI, et al. (2007) Shared risk and protective factors for overweight and disordered eating in adolescents. Am J Prev Med 33, 359-369.

32. MacKinnon DP (2008) Introduction to Statistical Mediation Analyses, 1st ed. New York: Lawrence Erlbaum Associates.

33. Conner M, Norman P \& Bell R (2002) The theory of planned behavior and healthy eating. Health Psychol 21, 194-201.

34. Ventura AK, Loken E, Mitchell DC, et al. (2006) Understanding reporting bias in the dietary recall data of 11-year-old girls. Obesity (Silver Spring) 14, 1073-1084. 